\title{
The Cognitive Enhancing Effect of Tomato Pomace
}

\author{
${ }^{1,2}$ Wipawee Thukhammee, ${ }^{1,2}$ Jintanaporn Wattanathorn, \\ ${ }^{1,2}$ Supaporn Muchimapura, ${ }^{3}$ Juntanee Uriyapongson, \\ ${ }^{1,2}$ Terdthai Tong-Un and ${ }^{1,2}$ Panakporn Wannanon \\ ${ }^{1}$ Department of Physiology, Faculty of Medicine, \\ ${ }^{2}$ Integrative Complementary Alternative Medicine Research Center, \\ ${ }^{3}$ Department of Food Technology, Faculty of Technology, \\ Khon Kaen University, Khon Kaen 40002, Thailand
}

Received 2012-02-22, Revised 2012-08-30; Accepted 2012-08-31

\begin{abstract}
Poor learning abilities and impaired memory are increasing their importance in accompany with the increased elderly population and stress exposure. Therefore, the development of cognitive enhancement is required. Recent findings showed that oxidative stress played the important role on the development of cognitive impairment in various conditions. Therefore the development of cognitive enhancer from vegetable possessing antioxidant is focused. The present study is carried out to determine the effect of dried tomato pomace powder on learning and memory both in normal and cognitive impairment conditions. Adult male Wistar rats, weighing 180-200 g, were orally given dried tomato pomace at various doses ranging from 2,10 and $50 \mathrm{mg} \mathrm{kg}^{-1} \mathrm{BW}$ at a period of 14 days. The animals were determined the cognitive enhancing effect of tomato pomace after single dose, 7 and 14 days after administration. The results showed that tomato pomace significantly decreased escape latency but increased retention time. In addition, we also determined the cognitive enhancing effect of this substance in cognitive deficit condition. In this part, all animals were induced cognitive deficit using scopolamine injection via intraperitoneal route after 14 days of treatment. Again, tomato pomace also improved cognitive impairment. Tomato pomace is the potential cognitive enhancer. However, the precise underlying mechanism is still required further investigation.
\end{abstract}

Keywords: Tomato Pomace, Learning and Memory, Cognitive Deficit, Scopolamine

\section{INTRODUCTION}

Poor learning abilities and impaired memory have been recognized as one important problem nowadays. Its importance is increasing with the increased elderly population and stress exposure. Therefore, the development of cognitive enhancer is required. Recent findings showed that oxidative stress played the important role on the development of cognitive impairment in various conditions including, aging mild cognitive impairment, Alzheimer's disease (Mecocci, 2004; Wattanathorn et al., 2010) chronic stress (Tishkina et al., 2009).

Up until now, many strategies have been developed to optimize learning and memory including exercise, dietary therapy, herbal therapy, pharmaceutical drugs and advanced medical device (Ingole et al., 2008). Among various strategies, dietary therapy such as functional food has gained much concentration. The application of food as medicine or functional food has been for more than 2500 years. It has been reported that food or every diet also offers health benefits and reduces the risk of chronic disease beyond the widely accepted nutritional effects. Functional foods are not pills or capsules but they are consumed as part-of a normal everyday diet. The beneficial effect of functional food appears to relate to its bioactive ingredient such as substances containing antioxidant (Raghuveer and Tandon, 2009). Tomato or Lycopersicon Esculentum, a plant in the family of Solanaceae, is one of the most frequently consumed vegetables in Thailand and worldwide. It is a good source of potassium, folate and the vitamins A, C and E. In addition to its micronutrient benefits, tomato also contains valuable phytochemicals, including carotenoids such as lycopene and betacarotene. Moreover, it also contains polyphenols such as flavonoids especially quercetin and kepferol (Canene-Adams et al., 2005). It had been reported that lycopene, one of the active Khon Kaen 40002, Thailand Tel: +66-43-348394 Fax: +66-43-348394 
component in tomato, could attenuate memory impairment in diabetes mellitus by inhibiting acetylcholinesterase enzyme resulting in an elevation of acetylcholine leading to learning and memory improvement. In addition, it also decreased oxidative stress both in cerebral cortex and in hippocampus (Rodrguez et al., 2006).

Not only lycopene but also beta-carotene provides benefit for brain functions. Previous study demonstrated that beta-carotene could attenuate neuronal damage in cerebral cortex and could improve memory impairment in SAM8 (Rodrguez et al., 2006). Moreover, it also decreased neurotoxicity induced by glutamate (Lemus-Molina et al., 2009) and oxidative stress (Prasad et al., 2008).

Recent findings show that most of the tomato waste product or tomato pomace obtained from food industry processing contained skin, pulp and crushed seeds of tomato. This tomato waste product still contains lycopene and beta-carotene (Sabio et al., 2003). However, it was reported that beta-carotene was a susceptible component toward thermal degradation (Abushita et al., 2000). Based on the crucial role of oxidative stress on cognitive impairment and the active ingredients possessing antioxidant in tomato pomace, we hypothesized that the dried powder containing lycopene could enhance memory in both normal healthy and cognitive impairment conditions. To test this hypothesis, the present study was designed to determine the effect of dried tomato pomace powder on learning and memory both in normal and cognitive impairment conditions.

\section{MATERIALS AND METHODS}

\subsection{Preparation of Dried Tomato Tomace Powder}

Tomato tomace is consisting of tomato skins, pulp and crushed seeds that remain after the processing of tomatoes for juice. Wet tomato pomace was dried with oven at 50 degree Celsius for $2 \mathrm{~h}$. The \%yield of tomato pomace is 12.75 . The dried tomato tomace was grinded as powder and determined both lycopene level and total phenolic compound and used as markers for quality control. It was found that the dried tomato tomace contained lycopene $160.94 \mathrm{mg} / 100 \mathrm{~g}$ sample while contained total phenolic compound $413.07 \pm 34.45 \mathrm{mg}$ of gallic acid equivalent $/ \mathrm{mg}$. The dried tomato pomace was kept in airtight container at room temperature until use. The administration of dried tomato tomace was performemed using propylene glycol as vehicle.

\subsection{Animals}

Adult male Wistar rats (180-200 g, 8 weeks old) were obtained from Animal Unit, Faculty of Medicine, Khon
Kaen University and were housed in group of 5 per cage in standard metal cages at $22 \pm 2^{\circ} \mathrm{C}$ on $12: 12 \mathrm{~h}$ light-dark cycle. All animals were given access to food and water ad libitum. The experiments were performed after the approval of protocol by the Ethical Committee of the Institution and every effort was made to minimize animal suffering in accordance with the internationally accepted principles for laboratory use and care of European Community (EEC directive of 1986; 86/609/EEC). The experimental protocols were approved by the institutional animal care and use committee.

\subsection{Experimental Protocol}

All rats were divided in to 7 groups as following; (1) control or naïve intact control (2) vehicle (propylene glycol) treated group (3) Aricept or donepezil $\left(1 \mathrm{mg} \mathrm{kg}^{-1} \mathrm{BW}\right)$ (4) Vitamin C (250 mg kg-1 BW) 5)-7) dried tomato pomace at various doses ranging from 2,10 and $50 \mathrm{mg} \mathrm{kg}^{-1} \mathrm{BW}$. In this study, Aricept or donepezil, a standard drug used for dementia treatment, is used as positive control. In addition to Aricept, vitamin C, a well known antioxidant possessing cognitive enhancing effect is also used as positive control. All rats had been treated with the assigned substance for 14 days. The cognitive enhancing effect was determined by evaluating the effect of dried tomato tomace on spatial memory using Morris water maze test. The escape latency and retention time in this test were used as indices for cognitive enhancing effect. The evaluation was performed after single dose, 7 and 14 days of administration. Then 24 $\mathrm{h}$ after the last evaluation, the rats were orally given the assigned treatment and scopolamine at dose of $3 \mathrm{mg} \mathrm{kg}^{-1}$ BW was administered via intra-peritoneal route $30 \mathrm{~min} 0$ before the assessment of spatial memory.

\subsection{Morris Water Maze Test}

The water maze consisted of a metal pool $(170 \mathrm{~cm}$ in diameter $\times 58 \mathrm{~cm}$ tall $)$ filled with tap water $(25, \mathrm{C}, 40 \mathrm{~cm}$ deep) divided into 4 quadrants. In the center of 1 quadrant was a removable escape platform below the water level and covered with a nontoxic milk powder. The pool was divided into 4 quadrants (NE, NW, SE and SW) by two imaginary lines crossing the center of the pool. For each animal, the location of invisible platform was placed at the center of one quadrant and remained there throughout training. The rats must memorize the platform location in relation to various environmental cues because there was nothing directly showed the location of the escape platform in and outside the pool. Therefore, the placement of the water tank and platform were the same in all acquisition trials. Each rat was gently placed in the water facing the wall of the pool from one of the four starting points ( $\mathrm{N}, \mathrm{E}, \mathrm{S}$ or $\mathrm{W}$ ) along 
the perimeter of the pool and the animal was allowed to swim until it found and climbed onto the platform. During training session, the rat was gently placed on the platform by experimenter when it could not reach the platform in $60 \mathrm{~s}$. In either case, the subject was left on the platform for $15 \mathrm{~s}$ and removed from the pool. The time for animals to climb on the hidden platform was recorded as escape latency or acquisition time. In order to determine the capability of the animals to retrieve and retain information, the platform was removed $24 \mathrm{~h}$ later and the rat was released into the quadrant diagonally opposite to that which contained the platform. Time spent in the region that previously contained the platform was recorded as retention time. In each trial, the animal was quickly dried with towel before being returned to the cage. All tests were carried out within $30 \mathrm{~min}$ after the administration of the assigned substance.

\subsection{Statistical Analysis}

Data are expressed as means \pm S.E.M. and were analyzed statistically by one-way ANOVA, followed by Post-hoc (LSD) test. The results were considered statistically significant at $\mathrm{P}$-value $<0.05$.

\section{RESULTS}

The effect of dried tomato pomace on the escape latency in Morris water maze test was shown in Fig. 1. The results showed that vehicle produced no significant change on escape latency. After single administration, aricept and dried tomato pomace treated group at dose of $2 \mathrm{mg} \mathrm{kg}^{-1} \mathrm{BW}$ significantly decreased escape latency (pvalue $<0.001$ and 0.01 respectively; compared to vehicle treated group) while no significant changes were observed in vitamin $\mathrm{C}$ treated group and dried tomato pomace treated group at dose of 10 and $50 \mathrm{mg} \mathrm{kg}^{-1}$. However, at 7 days of treatment, both Aricept and vitamin $C$ treated group showed significant decreased in escape latency ( $p$-value $<0.001$ all; compared to vehicle treated group). The dried tomato pomace treated groups at doses of 2,10 and $50 \mathrm{mg} \mathrm{kg}$ also significantly decreased escape latency ( $p$-value $<0.001 ; 0.01$ and 0.05 respectively; compared to vehicle treated group). When, the exposure time was prolonged to 14 days, both Aricept and vitamin $\mathrm{C}$ still showed significant reduction of escape latency ( $p$-value $<0.05$ and 0.01 respectively; compared to vehicle treated group). The rats treated with dried tomato pomace still showed the decreased escape latency however, the significant changes were observed only in the groups treated with dried tomato pomace at dose of 2 and $50 \mathrm{mg} \mathrm{kg}^{-1} \mathrm{BW}$ ( $p$-value $<0.01$ all; compared to vehicle treated group).

The effect of dried tomato pomace on the retention time was also determined and the results were shown in Fig. 2. It was found that the rats which received vitamin $\mathrm{C}$ and dried tomato pomace at doses of 10 and $20 \mathrm{mg}$ $\mathrm{kg}^{-1} \mathrm{BW}$ significantly enhanced retention time (pvalue $<0.001 ; 0.01$ and 0.05 respectively; compared to vehicle treated group). When the treatment was prolonged further to 7 and 14 days, the rats which received vitamin $\mathrm{C}$ still showed significant increased retention times ( $p$-value $<0.01$ and 0.05 respectively; compared to vehicle treated group). However, it was found that the dried tomato pomace treated groups at all dosage range used in this study significantly enhanced retention time at 7 days of treatment ( $p$-value $<0.05$; 0.001 and 0.05 respectively; compared to vehicle treated group). Unfortunately, we failed to observe the significant changes at 14 days of treatment.

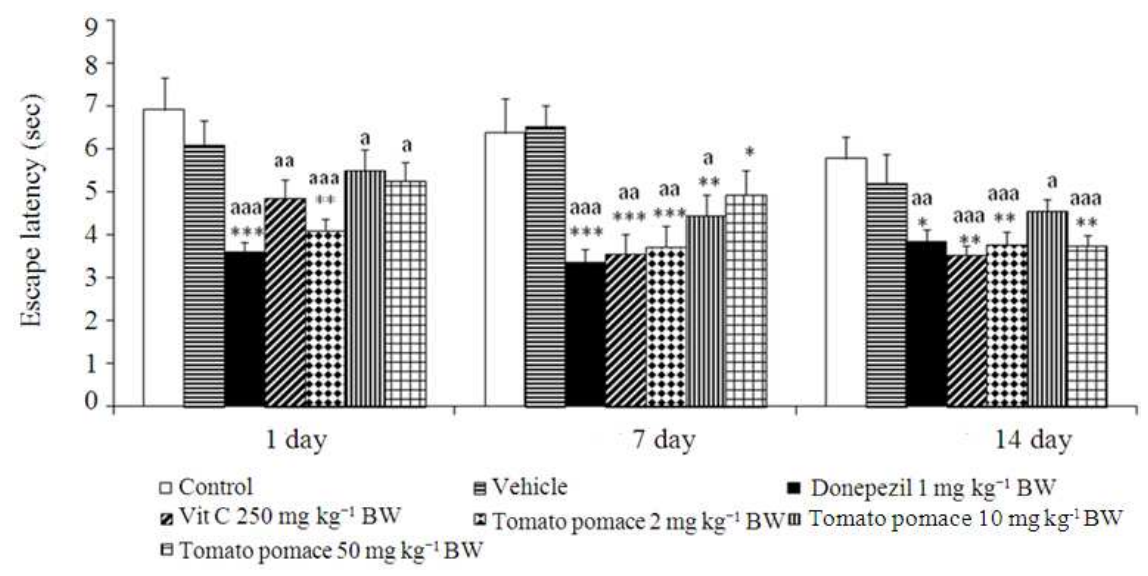

Fig. 1. Effect of dried tomato pomace on the escape latency of normal healthy rats in Morris Water Maze Test (MWT) (n=10/group) ${ }^{\text {a,aa,aaa }}$ value $<0.05 ; .01$ and 0.001 respectively; compared to naïve control group) $*, * *, * * *$ p-value $<0.05 ; .01$ and 0.001 respectivel 


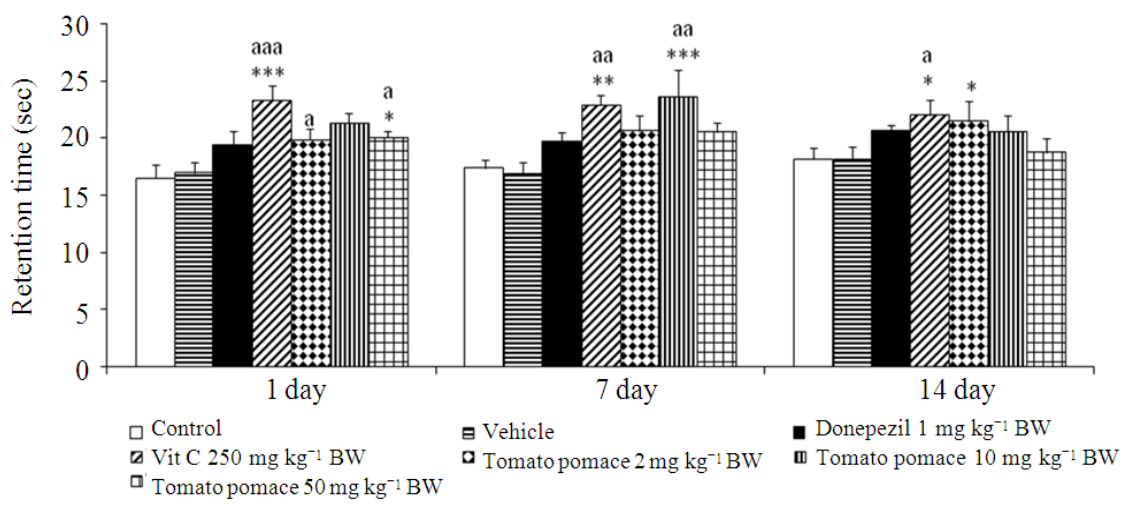

Fig. 2. Effect of dried tomato pomace on the retention time of normal healthy rats in Morris water maze test (MWT) (n=10/group) a,aa,aaa p-value $<0.05 ; 0.01$ and 0.001 respectively; compared to naïve control group) ${ }^{*, * * * * * *}$-value $<0.05 ; .01$ and 0.001 respectively; compared to vehicle treated group)

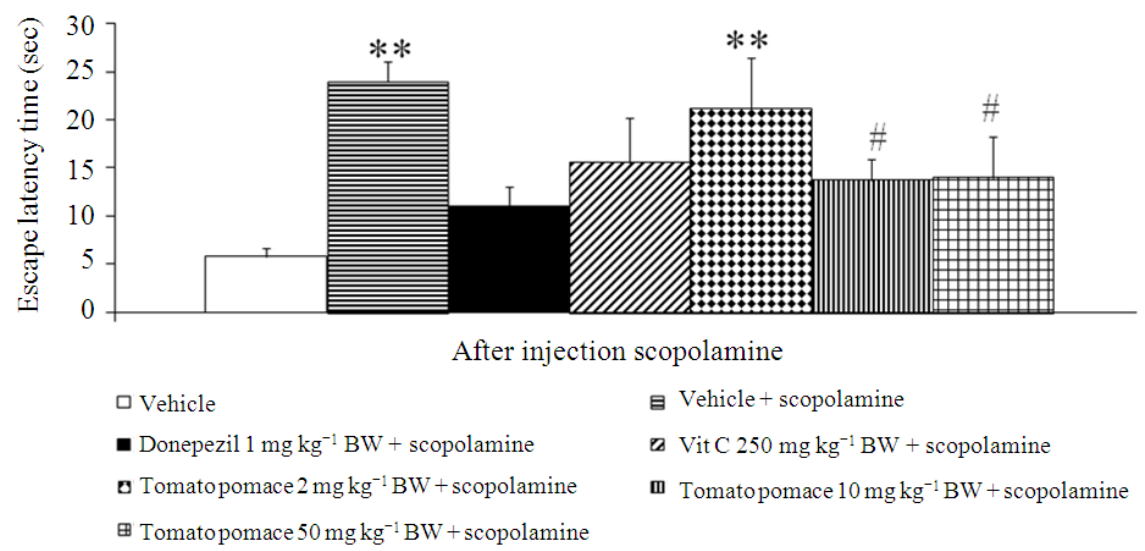

Fig. 3. Effect of dried tomato pomace on the escape latency of cognitive deficit rats in Morris Water Maze Test (MWT) ( $n=10 /$ group) ${ }^{* *} \mathrm{p}$-value $<0.01$; compared to vehicle treated group) $\# \mathrm{p}$-value $<0.05$; compared to vehicle plus scopolamine

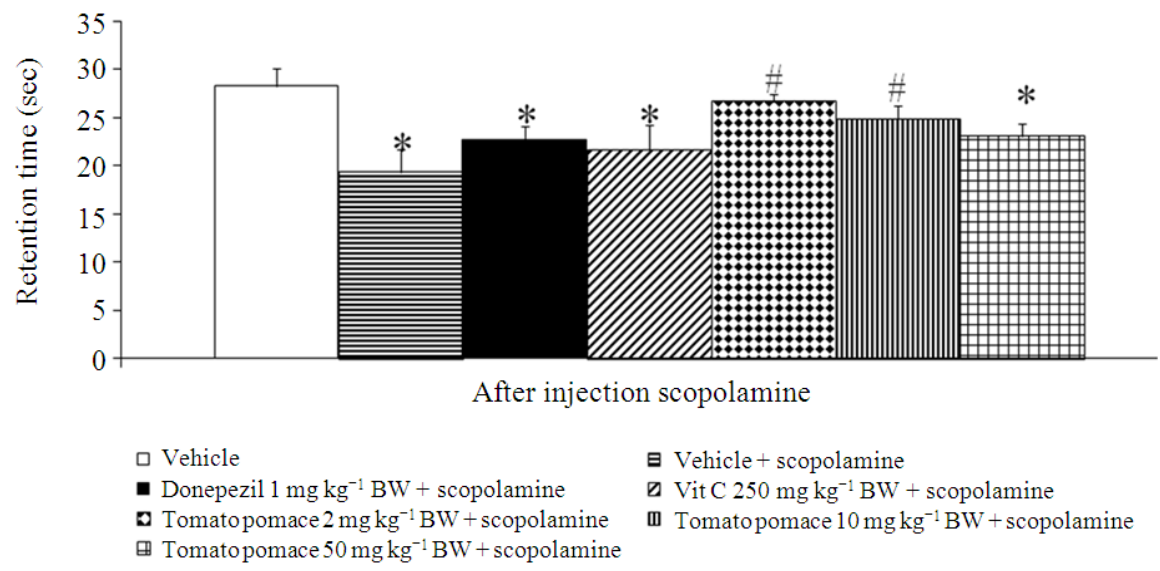

Fig. 4. Effect of dried tomato pomace on the retention time of cognitive deficit rats in Morris Water Maze Test (MWT) (n=10/group) ${ }^{*}$ p-value $<0.05$; compared to vehicle treated group) \#p-value $<0.05$; compared to vehicle plus scopolamine 
In addition to the determination of cognitive enhancing effect of dried tomato pomace in normal healthy condition, we also determined the cognitive enhancing effect of dried tomato pomace in cognitive deficit condition induced by scopolamine. Figure 3 showed that the animals which received vehicle plus scopolamine significantly enhanced escape latency time ( $p$-value $<0.01$; compared to vehicle treated group). The rats which received dried tomato pomace at dose of 10 and $50 \mathrm{mg} \mathrm{kg}^{-1} \mathrm{BW}$ significantly attenuated the elevation in escape latency induced by scopolamine ( $p$-value $<0.05$ all; compared to vehicle treated group). The current results also showed that rats which received vehicle plus scopolamine significantly decreased retention time ( $p$ value $<0.001$; compared to vehicle treated group). It was found that dried tomato pomace at doses of 2 and $10 \mathrm{mg}$ $\mathrm{kg}^{-1} \mathrm{BW}$ significantly reversed retention time (pvalue $<0.05$; compared to vehicle plus scopolamine treated group) as shown in Fig. 4.

\section{DISCUSSION}

The present study clearly demonstrates that dried tomato pomace can enhance spatial memory both in healthy and cognitive deficit conditions.

Learning has been defined as the process acquiring the knowledge while memory is the retention of the acquired knowledge that can be retrieved (Kandel et al., 2000). Our results showed that under normal condition, dried tomato pomace could exert its influence on both escape latency and retention time. Therefore, it indicated that it could influence on both learning acquisition, consolidation and retrieval processes. Since the pattern of changes of escape latency induced by dried tomato pomace was similar to those of Aricept and vitamin $\mathrm{C}$, we suggested that dried tomato pomace might exert its effect to facilitate acquisition phase partly via the alteration of neurotransmitter such as acetylcholine and via its antioxidant effect. However, only viamin $\mathrm{C}$ that showed the same pattern of changes similar to that of dried tomato pomace. Therefore, its effect to enhance consolidation and retrieval process might be related to its antioxidant activity. Recent findings showed that consolidation process involved protein synthesis (Bermudez-Rattoni, 2010). Previous study revealed that oxidative stress could disturb the function of protein contributing important role on consolidation process and resulting in memory impairment (Achat-Mendes et al., 2007). Therefore, the antioxidant effect of dried tomato pomace might attenuate the disturbance of protein molecules induced by oxidative stress.
In cognitive deficit condition, the escape latency changes induced by dried tomato pomace showed the same pattern as vitamin $C$. Therefore, tomato pomace might exert its effect via its antioxidant. In contrast to the changes of escape latency, the retention time of tomato pomace demonstrated the similar patterns as those observed after treatment with Aricept and vitamin C. Therefore, the tomato pomace's effect on retention time in cognitive deficit condition might relate in part to available acetylcholine and oxidative stress.

Taken all data together, it was possible that dried tomato pomace might exert its influence on acquisition phase in normal condition differ from that in cognitive deficit condition. However, its influence on retention in normal condition might occur via its antioxidant while this effect in cognitive deficit conditions appeared to occur partly via both the available of acetylcholine and its antioxidant.

\section{CONCLUSION}

Tomato pomace can serve as potential cognitive enhancer both in normal and cognitive deficit conditions. Thus, the development tomato pomace as functional food is very much interesting because it not only provides health benefit but also increases its value. However, the precise underlying mechanism is still required further researches.

\section{ACKNOWLEDGEMENT}

This study was supported by National Research Council of Thailand, The Integrative Complementary and Alternative Medicine Research center. The Royal Golden Jubilee Ph. D Program Thailand.

\section{REFERENCES}

Abushita, A.A., H.G. Daood and P.A. Biacs, 2000. Change in carotenoids and antioxidant vitamins in tomato as a function of varietal and technological factors. J. Agric. Food Chem., 48: 2075-2081. PMID: 10888501

Achat-Mendes, C., K.L. Anderson and Y. Itzhak, 2007. Impairment in consolidation of learned place preference following dopaminergic neurotoxicity in mice is ameliorated by $\mathrm{N}$-acetylcysteine but not D1 and D2 dopamine receptor agonists. Neuropsychopharmacology, 32: 531-541. PMID: 16760923 
Bermudez-Rattoni, F., 2010. Is memory consolidation a multiple-circuit system? Proc. Natl. Acad. Sci. USA., 107: 8051-8052. DOI: 10.1073/pnas.1003434107

Canene-Adams, K., J.K. Campbell, S. Zaripheh, E.H. Jeffery and J.W. Erdman Jr., 2005. The tomato as a functional food. J. Nutr., 135: 1226-1230. PMID: 15867308

Kandel, E.R., J.H. Schwartz and T.M. Jessell, 2000. Principles of Neural Science. 4th Edn., McGrawHill, Cambridge, ISBN-10: 0838577016, pp: 1414.

Lemus-Molina, Y., M.V. Sanchez-Gomez, R. DelgadoHernandez and C. Matute, 2009. Mangifera indica L. extract attenuates glutamate-induced neurotoxicity on rat cortical neurons. Neurotoxicology, 30: 10531058. PMID: 19591864

Mecocci, P., 2004. Oxidative stress in mild cognitive impairment and Alzheimer disease: A continuum. J. Alzheimer's Dis., 6: 159-163. PMID: 15096699

Prasad, S., N. Kalra, M. Singh and Y. Shukla, 2008. Protective effects of lupeol and mango extract against androgen induced oxidative stress in Swiss albino mice. Asian J. Androl., 10: 313-318. PMID: 18097535

Rodrguez, J., D.D. Pierro, M. Gioia, S. Monaco and R. Delgado et al., 2006. Effects of a natural extract from Mangifera indica L and its active compound, mangiferin, on energy state and lipid peroxidation of red blood cells. Biochim. Biophys. Acta, 1760: 13331342. PMID: 16860486
Raghuveer, C. and R.V. Tandon, 2009. Consumption of and our health concern. Pak. J. Physiol., 5: 76-83.

Sabio, E., M. Lozano, V.M.D. Espinosa, R.L. Mendes and A.P. Pereira et al., 2003. Lycopene and $\beta$ carotene extraction from tomato processing waste using supercritical $\mathrm{CO}_{2}$. Ind. Eng. Chem. Res., 42: 6641-6646. DOI: 10.1021/ie0301233

Ingole, S.R., K.S. Rajput and S.S. Sharma, 2008. Cognition enhancers: Current strategies and future perspectives. CRIPS, 9: 42-48.

Tishkina, A.O., I.P. Levshina, N.A. Lazareva, N.V. Passikova and M.Y. Stepaniche et al., 2009. Chronic stress induces nonapoptotic neuronal death in the rat hippocampus. Dokl Biol. Sci., 428: 403-406. PMID: 19994774

Wattanathorn, J., S. Machimapura, T. Tong-Un and W. Phachonpai, 2010. Nasal administration of quercetin liposomes improves memory impairment and neurodegeneration in animal model of Alzheimer's disease. Am. J. Agric. Biol. Sci., 3: 286-293. DOI: 10.3844/ajabssp.2010.286.293 